\title{
Study on the Calibration Optimization of the Laser Lidar for WFCTA of LHAASO
}

\section{Fengrong Zhu *i}

School of Physical Science and Technology, Southwest Jiaotong University, Chengdu 610031,

P.R. China, E-mail: zhufr@home.swjtu.edu.cn

\section{Yong Zhang}

Key Laboratory of Particle Astrophysics, Institute of High Energy Physics, Chinese Academy of Science, P.O. Box 918, 100049 Beijing, China

\section{Ning Xie}

School of Physical Science and Technology, Southwest Jiaotong University, Chengdu 610031, China

\section{H.Y. Jia,}

School of Physical Science and Technology, Southwest Jiaotong University, Chengdu 610031, China

\section{X.M. Li,}

School of Physical Science and Technology, Southwest Jiaotong University, Chengdu 610031, China

\begin{abstract}
Wide Field view Cherenkov Telescope Array(WFCTA) of LHAASO, consisting of 12 telescopes, has been proposed to mounted at Daocheng County, Sichuan Province, China, to probe the galactic cosmic rays with the ultra energy of $3 \times 10^{13} \mathrm{eV}-10^{17} \mathrm{eV}$ together with WCDA and KM2A of LHAASO. 4 laser system and 2 WFCTA prototypes will be built to calibrate the WFCTA. Considering the Rayleigh scattering and propagation together with aerosol scattering, ozone absorbtion and propagation of the laser in the air, the response of WFCTA prototypes to the light, the optimization scheme of the calibration, procedure and results based on rays tracing method will be talked in detail in this presentation.
\end{abstract}

35th International Cosmic Ray Conference - ICRC 2017

10-20 July, 2017

Bexco, Busan, Korea

\footnotetext{
* Speaker.

${ }^{\dagger}$ on behalf of the LHAASO Collaboration.
} 


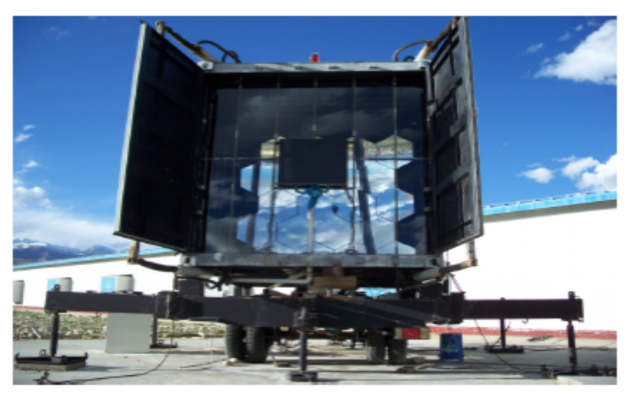

Figure 1: WFCTA prototype, including a reflecting mirror and a 256 PMTs array

\section{Introduction}

The Large High Altitude Air Shower Observatory (LHAASO) is a multi-objective project to probe the origin of cosmic rays with a complicated $1.2 \mathrm{~km}^{2}$ ground-based detector array[1]. The total three components are KM2A KM2A[2, 3] , WCDA[4] and WFCTA[5]. And KM2A is one kilometer square extensive air shower array, designed for the measurement of the number density and arrival time of shower particles, contains 5242 EDs (Electromagnetic particle Detectors) and 1221 MDs (Muon Detectors). WCDA, the water Cherenkov detector array, with an area of $78000 \mathrm{~m}^{2}$ and 350000 tons of purified water, is planned to be built in a couple of years at Mount Haizi, Daocheng, at an altitude of $4410 \mathrm{~m}$ a.s.l. The main purpose of the WCDA is to survey the northern sky for VHE gamma ray sources. WFCTA, consiting of 12 telescopes, has been proposed to mounted at Daocheng County, Sichuan Province, China. Its major goal is to probe the galactic cosmic rays with the ultra energy of $3 \times 10^{13} \mathrm{eV}-10^{17} \mathrm{eV}$ together with WCDA and KM2A of LHAASO.

The laser lidar calibration system for the WFCTA is to be built with a series of devices, mainly including two WFCTA prototypes, two YAG laser system, two nitrogen laser system with high precision revolving table, an infrared thermometer and a meteorological station. And it can study absolute calibration of photon number, air quality monitoring and back scatter method monitoring weather, also study the measurement of the horizontal attenuation length, distribution of aerosol scatter angle, aerosol effective scale height.

The Nitrogen laser system(LS) prototype had operated and acquired data for physics analysis on ARGO-YBJ site for about four years, which will be mounted at LHAASO site. The YAG LS will to be built at some site near LHAASO, seperately. In this proceeding the simulation for Nitrogen laser system and YAG laser system will be done. We will simulate the responses of WFCTA prototype vary with the site of laser LS and the direction of WFCTA prototype. The optimization scheme is introduced in Section 2 and the optimization results based on ray trace are presented in Section 3.

\section{Optimization scheme}

\section{1 detector configure}

To simplify the optimization procedure, the laser shot energy are set $170 \mu \mathrm{J}$ for $\mathrm{N} 2$ and $1.7 \mathrm{~mJ}$ 


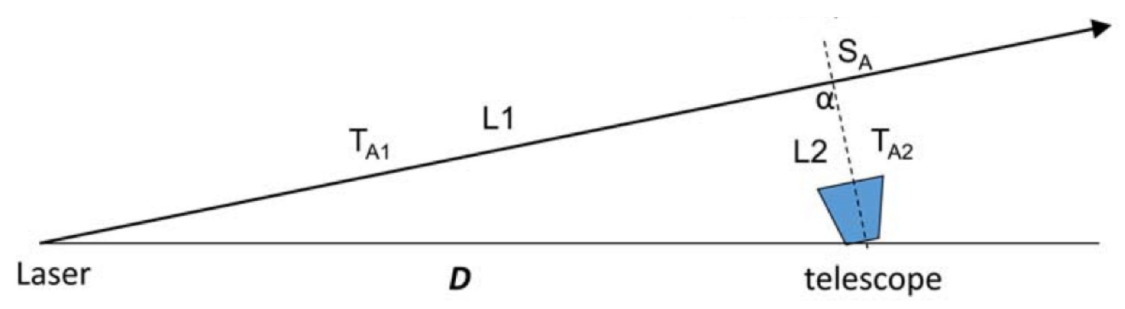

Figure 2: The absolute calibration geometry, Laser reprents the laser device and telescope is the WFCTA prototype

for YAG. The LS is thought to emit the laser only. As for the light receiver system as shown in Figure 1, the container enclusing the WFCTA telescope prototype has the size of $2.85 \mathrm{~m} \times 2.13 \mathrm{~m}$ $\times 2.18 \mathrm{~m}$. The reflecting mirror, 20 sub-mirrors with 6 regular hexagon, total $4 \mathrm{~m}^{2}$ with the curve radius $5.5 \mathrm{~m}$ and focal plane $2.5 \mathrm{~m}$. The camera to receive light, consists of $16 \times 16$ PMTs array, is about $1850 \mathrm{~cm}^{2}$. The transparency of all the sub-mirrors are the same, and quantum effciency of all the PMTs are also same. In order to decrease rayleish scattering effect according to[6], the sum of the zenith angles of laser device and WFCTA prototype are 90 degrees. Considering the field of view of telescope, the elevation angle of the reflectiong mirror are set from 10 to 80 degrees with step of 5 degrees. The distance between the telescope prototype and laser device are different for YAG and N2. It is from $3 \mathrm{~km}$ throught $11 \mathrm{~km}$ with step of $1 \mathrm{~km}$ for YAG and for $\mathrm{N} 2$ from $100 \mathrm{~m}$ to $2000 \mathrm{~m}$. The geometry of the absolute calibration is shown like in Figure 2. A shot photons emitted from Laser(N2 laser system or YAG Laser system) transport in the air, then light scattered in point $\mathrm{S}_{A}$ produces tracks in the WFCTA prototype.

\subsection{Physical processes selection during transportation of light}

The telescope prototype recevie the scattered light from laser shot with $10^{14} \sim 10^{15}$ photons. In order to save the consumption of simulation time, It is important to select the physical processes among the Rayleish scattering , aerosol scattering and Ozone absorption during transportation of light. Rayleigh scattering is the scattering of light by particles smaller than its wavelenth. Molecules in the atmosphere predominantly scatter, rather than absorb, light emitted from Laser. These light also suffers Mie scattering throught interaction with small dust particles suspended in the air(aerosols), whose size is comparable to the wavelength of the light. In our simulation of the attenuation due to aerosols we have used the model proposed by Elterman[7,8]. The photons are absorped by Ozone in the air following the Elterman standard atmosphere[8]. Rayleish scattering, Mie scattering and Ozone absorption processes can be treated separately. In the following, the term "attenuation" is used to indicate photons that are scattered in such a way that they do not contribute to the light signal recorded by the telescope. The molecular, aerosol attenuation processes and ozone absorption processes can be described in terms of atmospheric transmission coefficients $\mathrm{T}_{m o l}(\lambda, \mathrm{s}), \mathrm{T}_{a e r}(\lambda, \mathrm{s})$ and $\mathrm{T}_{o z}(\lambda, \mathrm{s})$, indicating the fraction of transmitted light intensity as a function 


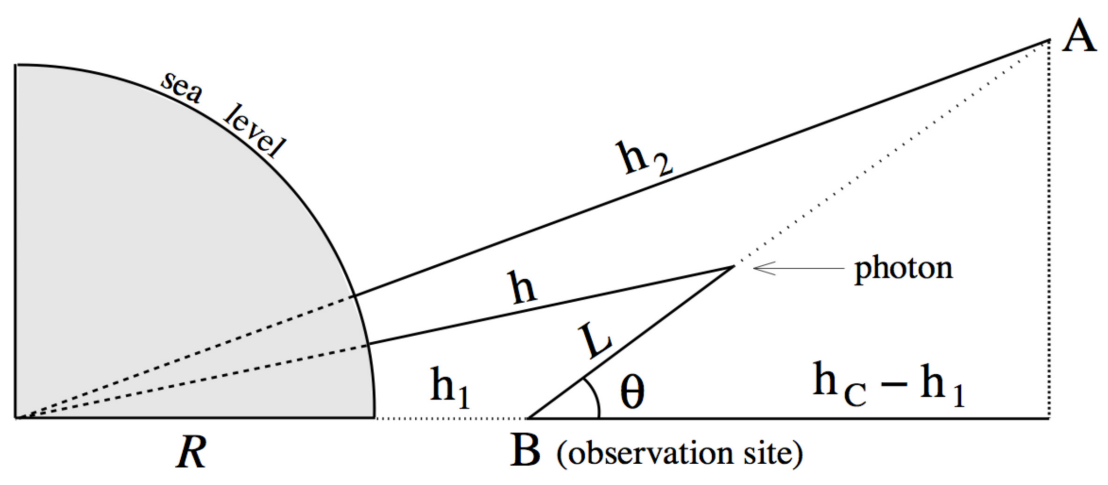

Figure 3: Calculation of the true vertical height $\mathrm{h}_{2}$ of the scatted point of light(point $\mathrm{A}$ ), and the optical path traversed down to the telescope (point B).

of the wavelength $\lambda$ and the path length s. The amount of light recorded at the telescope prototype aperture $\mathrm{I}(\lambda, \mathrm{s})$ can be expressed in terms of the light intensity at the source $\mathrm{I}_{0}(\hat{\mathrm{I}} \dot{z}, \mathrm{~s})$ as

$$
T(\lambda, s)=I_{0}(\lambda, s) \cdot T_{m o l}(\lambda, s) \cdot T_{a e r}(\lambda, s) \cdot T_{o z}(\lambda, s) \cdot(1+H . Q .) \frac{d \Omega}{4 \pi}
$$

where H.O. are higher order corrections due to multiple scattering and $\mathrm{d} \Omega$ is the solid angle subtended by the telescope aperture as seen from the light source.

The transimission coefficient due to Rayleish scattering can be determined analytically by the equation:

$$
I(\lambda, s)=\exp \left[-\frac{I\left(\theta, h_{1}, h_{2}\right)}{x_{R}}\left(\frac{400 n m}{\lambda}\right)^{4}\right]
$$

Here $I\left(\theta, h_{1}, h_{2}\right)$ is the optical path $\left(\right.$ in $\left.\mathrm{g} / \mathrm{cm}^{2}\right)$ traversed between point $\mathrm{A}$ and point $\mathrm{B}$, and $\mathrm{x}_{R}=$ $2970 \mathrm{~g} / \mathrm{cm}^{2}$ is the mean free path of the Rayleigh scattering.

The aerosol attenuation coefficients $T_{a e r}(\lambda, s)$ in the Elterman model for the trajectory from A to $\mathrm{B}$ is :

$$
T_{m o l}(\lambda, s)=\exp \left(-\tau_{\text {aer }}\right), \tau_{\text {aer }}\left(h_{1}, h_{2}, \theta, \lambda\right)=\frac{\beta_{p}(0, \lambda)}{N_{p}(0)} \int_{h_{1}}^{h_{2}} N_{p}(h) \frac{d L}{d h} d h
$$

Here $\tau_{\text {aer }}$ is the aerosol opetical depth of the path from A to B as shown in Figure 3. Here R is the Earth radius, $\mathrm{h}_{1}$ the height(a.s.l) of the observation level and $\theta$ is the zenith angle of the photon trajectory measured at the telescople site. $N_{p}$ is the aerosol number density which roughly decreases exponetially up to $10 \mathrm{~km}$ a.s.l. with scale height of about $1.2 \mathrm{~km}$, followed by a more tenuous layer between 10 and $30 \mathrm{~km} . \beta_{p}(0, \lambda)$ is measured values of the aerosol attenuation coefficients at sea level, and we simply do $\beta_{p}(h, \lambda)=\beta_{p}(0, \lambda) \cdot N_{p}(h) / N_{p}(0)$.

The transmission coefficient of Ozone from A throught B is then:

$$
T_{o z}(\lambda, s)=\exp \left(-\tau_{o z}\right), \tau_{o z}\left(h_{1}, h_{2}, \theta, \lambda\right)=A_{v}(\lambda) \int_{h_{1}}^{h_{2}} D_{3}(h) \frac{d L}{d h} d h
$$




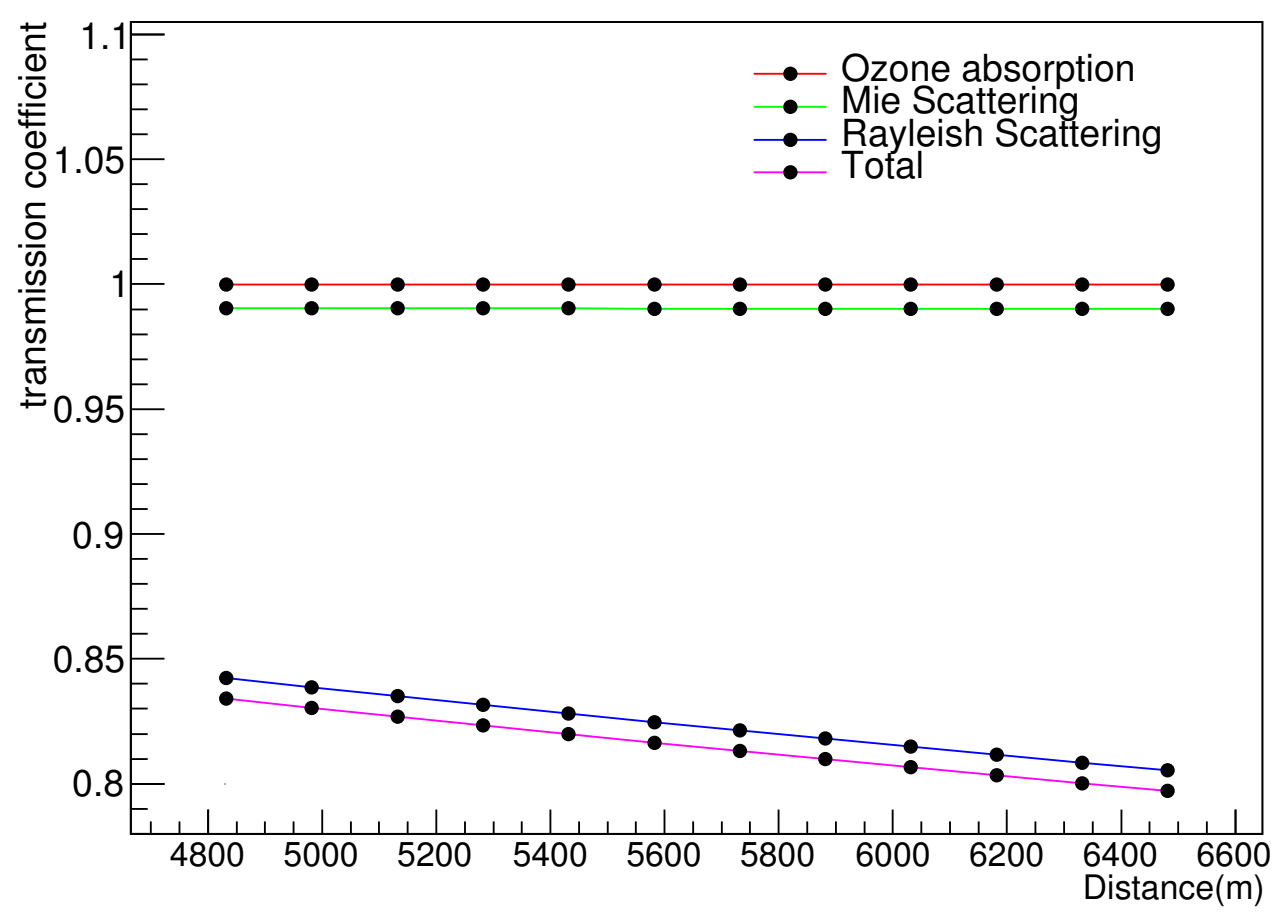

Figure 4: Transmission coefficient as a fucntion of the distance of light emitted from LS to LS, the horizontal axis gives the distance between the light emitted from LS and LS in the field of view of WFCTA prototype, and the vertical axis is transmission coefficient at different distance for Ozone absorption(red), Mie scattering(green), Rayleish scattering(blue) and total transmission coefficient(purple).

where $\tau_{o z}$ is the optical depth, $A_{v}(\lambda)$ is Vigroux ozone absorption coefficient $\left(\mathrm{cm}^{-} 1\right)$ and $D_{3}(h)$ is the ozone concentration $\left(\mathrm{cm} \mathrm{km}^{-} 1\right)$.

Finally, the overall atmospheric transmission coefficient is calculated as

$$
T_{\text {total }}=T_{m o l} \cdot T_{a e r} \cdot T_{o z}
$$

for our case, test beam light wavelength is $350 \mathrm{~nm}$ and $375 \mathrm{~nm}$, the distance between the telescope prototype and LS is not more far than $12 \mathrm{~km}$, the Rayleish scattering turns out to be dominant among three attenuation processes as shown in Figure 4, and ratio of Mie scattering effect to all is $1 \sim 2 \%$ and Ozone absorption effect $0.2 \%$, so the number of scattered light in the two processes toward telescope prototype is much fewer than in Rayleish scattering . It is reasnable to take only Rayleish scattering into consideration in our calibration optimization study of WFCTA. The number of scattered light in Raylesih scattering follows the law of $1+\cos ^{2} \theta, \theta$ is the scattered angle.

\subsection{Simplified simulation approach}

A full simulation should be the best approach for calculating the performance of each configuration, but in practice, it would be exhausted due to the fact that the simulation procedure is quite 


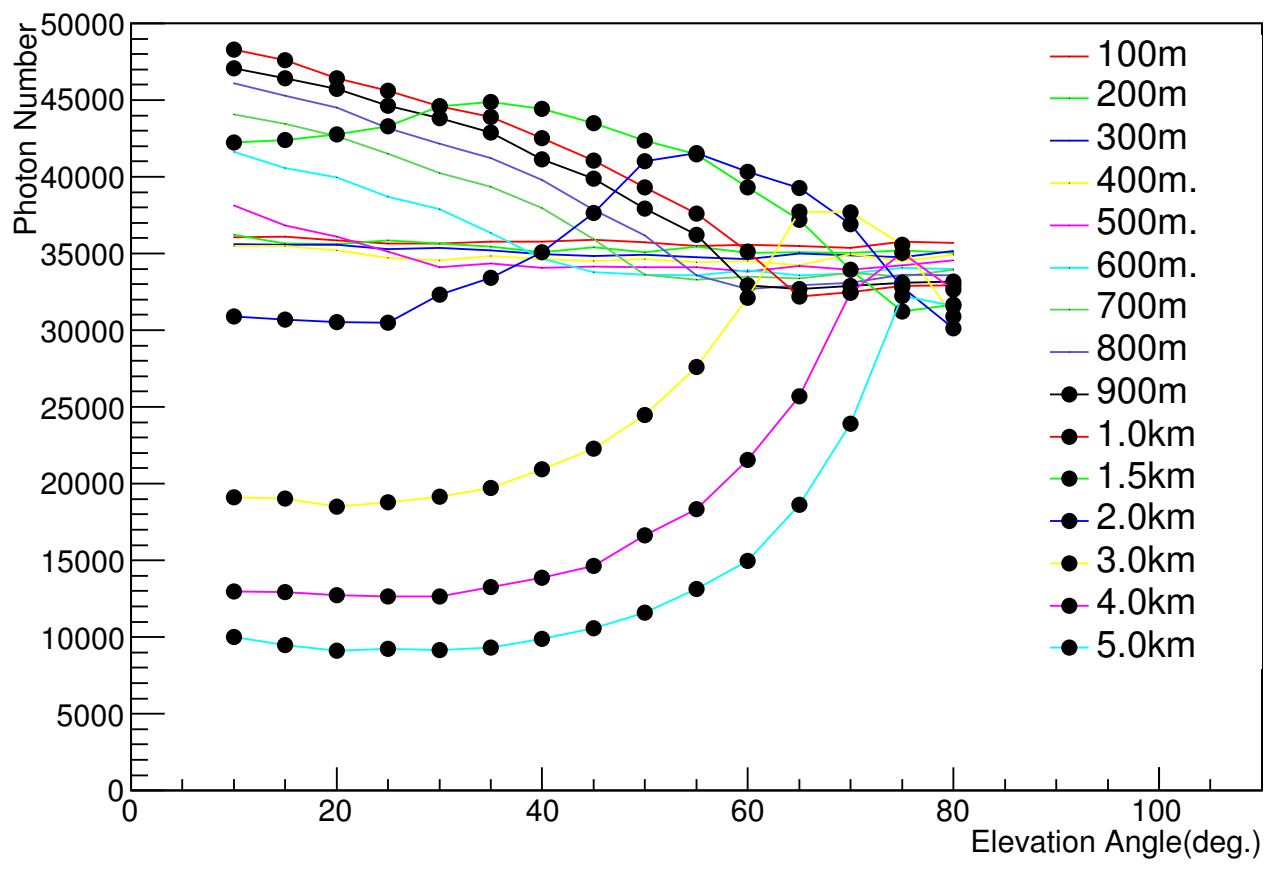

Figure 5: The number of the photons detected during one laser shot VS. elevation angle of the reflectiong mirror of the WFCTA prototype

time-consuming, especially the process of tracing of the lights in the air. a simple and efficient optimization procedure is proceeded.

First of all, in order to lessen the burden of iterations in the simulation, we trace the quantity of the attenuation in laser shot with step of $1 \mathrm{~m}$, not one photon by one photon, while we sample the scattered photons from 80 to 100 degree of scattered angle range and trace them one by one.

\section{3. simulation results}

\subsection{N2 laser simulation results}

Figure 5 shows the number of the photons detected by WFCTA telescope prototype varies with the elevation angle of the reflecting mirror, which is the zenith angle of principle axis of the reflecting mirrors. Different colors reprent the different distance between the laser device and the WFCTA prototype from $100 \mathrm{~m}$ to $5.0 \mathrm{~km}$ as shown in the figure. All these distances should meet the calibration requirement because the electronics of WFCTA prototype can accept the number of photons from several to several hundred thousands. For the distance from $100 \mathrm{~m}$ to $400 \mathrm{~m}$, all the elevation angles from 10 to 80 degrees the number of the photon detected change smaller corresponding to other cases, so these distance with elevation angles for the calibration should be the best in practice. Some elevation angles can be adopted for the distance from $500 \mathrm{~m}$ to $1 \mathrm{~km}$, but the distance from $1.5 \mathrm{~km}$ to $2.0 \mathrm{~km}$ should not be used because of their more changes in the number of the photons detected with elevation angle. For the distances from $3 \mathrm{~km}$ to $5 \mathrm{~km}$, the 


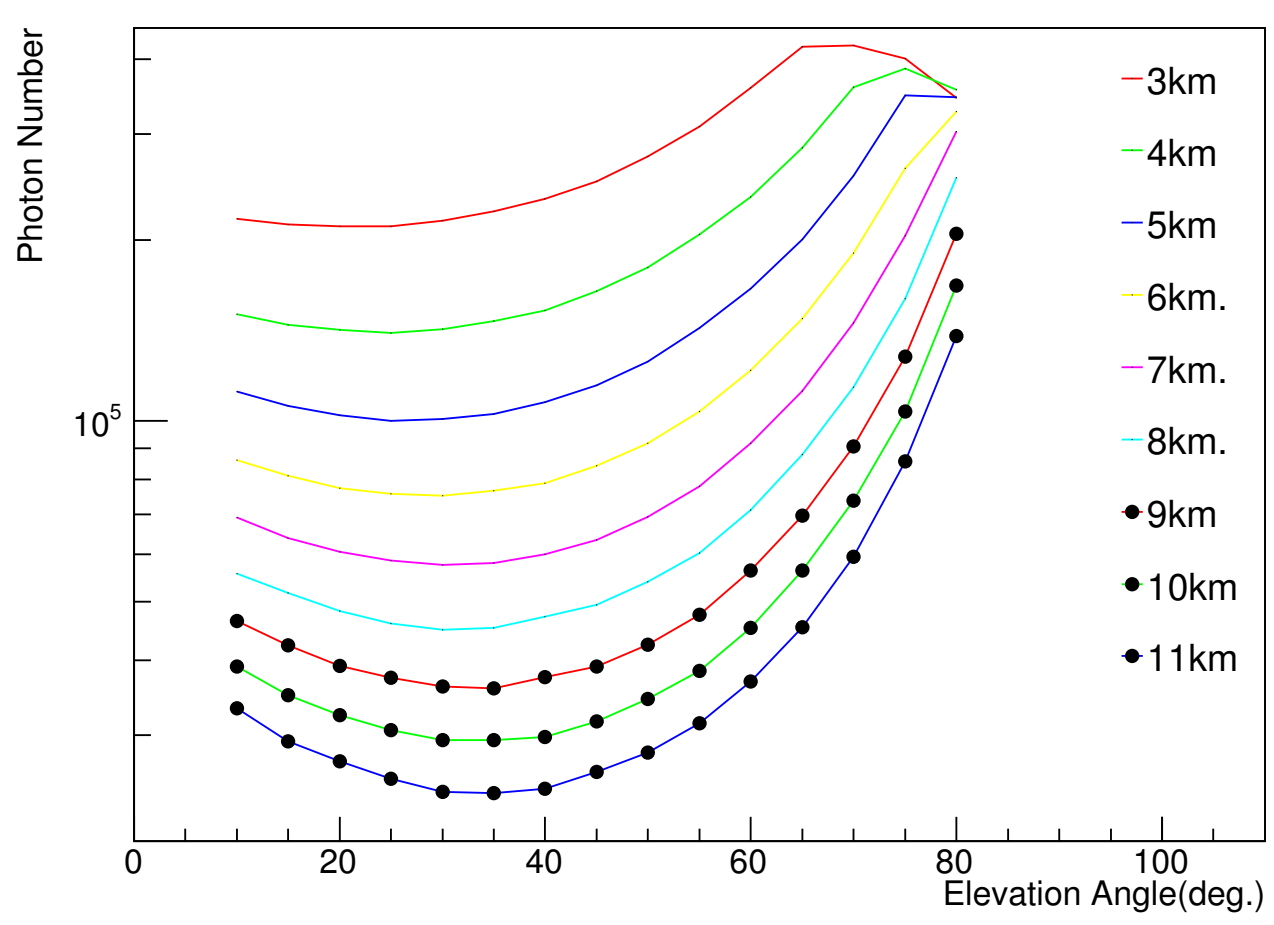

Figure 6: The number of the photons detected during one laser shot VS. the elevation angle of the reflecting mirror

photon number changes smaller when elevation angle is less than 40degree, so these distance with elevation angles configuration should also be better for our calibration.

\subsection{YAG laser simulation results}

Compared with N2 laser shot YAG laser shot has high energy of 10 times of the N2 laser shot , it can be placed more far away from the telescope to meet the electronics of WFCTA prototype. As shown in Figure 6, the number of photon detected by detector will be bigger than one hundred thousand for the distance from $3 \mathrm{~km}$ to $5 \mathrm{~km}$ with all the direction of the refelcting mirror, so these distances are too near for the WFCTA prototype. For the distance from $6 \mathrm{~km}$ to $11 \mathrm{~km}$, the photon number firstly decrease slowly with elevation angle, then reach the minimum near 35 degree of elevation angle, after 40 degree the photon number increase quickly with the elevation angle. So it is feasible for YAG laser calibration to locate the laser device far away from the telescope $6 \mathrm{~km}$ to $11 \mathrm{~km}$ or beyond, but the elevation angle of the reflecting mirrors of WFCTA prototype should be set near the 35 degree.

\section{4. summary and discussion}

In the calibration optimization of the laser lidar for WFCTA of LHAASO, firstly we calculated the transmission coefficient for the laser by molecule, aerosol and ozone in the air, and concluded 
that the Rayleish scattering effect is the most significant during the transporting process for our calibration study. For the N2 laser shot we reached that the distrance between the reflecting mirror and laser LS should be less $500 \mathrm{~m}$ or greater than $1.5 \mathrm{~km}$ with the elevation angle less than 30 degree. For the YAG laser the distance between the reflecting mirrors and laser device should be great $6 \mathrm{~km}$ with the elevation angle near 35 degree of the reflecting mirror.

The studies on the configuration of the calibration system are the preliminary results, the check is going well on. Also in the transporting process the mutiple scattering effect to laser in the air has not been taken into consideration, we will study it in our next plan althought it is smaller in our scattering and transporting process. The simulation on the scaler height of aerosol and other issues correlated with calibration of WFCTA will be done in the future.

\section{Knowledgement}

This work is supported by National Natural Science Foundation (NSFC) of China under contacts No. 11635011 and 11205126.

\section{References}

[1] Cao, Z. (for the LHAASO Collaboration) 2010, Chin. Phys. C, 34, 249

[2] Xu Wang, Zhong-Quan Zhang, Ye Tian et al. 2016, Chin. Phys. C, 40(8),86003

[3] ZHAO Jing;LIU Jia;SHENG Xiang-Dong et al. 2014, Chin. Phys. C, 38(11),36002

[4] Cong Ma, Lei Zhao, Yu-Xiang Guo, Jian-Feng Liu, Shu-Bin Liu, Qi An. 2016, Chin. Phys. C,40 (1) 16101

[5] MA Ling-Ling;BAI Yun-Xiang;CAO Zhen et al. 2011, Chin. Phys. C,35(5) 478

[6] Tracey Tessier et al for the HiRes Collaboration, ICRC 1995,5

[7] L. Elterman, 1964, Applied Optics Vol. 3, No. 6,745.

[8] L. Elterman, R.B. Toolin, S.L. Valley,1965, Handbook of geophisics and space environments, McGraw-Hill, N.Y. 